\title{
Fatores que podem contribuir para recessão gengival na clínica odontopediátrica: revisão narrativa de literatura
}

\author{
Factors that can contribute to gingival recession in pediatric dentistry: narrative literature review \\ Factores que pueden contribuir a la recesión gingival en odontología pediátrica: revisión narrativa \\ de la literatura
}

Recebido: 28/10/2021 | Revisado: 07/11/2021 | Aceito: 08/11/2021 | Publicado: 13/11/2021

Karine Fausto Gonzalez
ORCID: https://orcid.org/0000-0001-7131-0821
Universidade Metropolitana de Santos, Brasil
E-mail: karinefaustogonzalez@ gmail.com
Ariane Abdala Santana
ORCID: https://orcid.org/0000-0002-1159-7223
Universidade Metropolitana de Santos, Brasil
E-mail: arianeabdala.cd@ gmail.com
Keller de Martini
ORCID: https://orcid.org/0000-0002-7894-3207
Universidade Metropolitana de Santos, Brasil
E-mail: kellermartin1999@ gmail.com
Karla Mayra Rezende
ORCID: https://orcid.org/0000-0003-4340-0699
Universidade de São Paulo, Brasil
Universidade Metropolitana de Santos, Brasil
E-mail: karla.rezende@ usp.br

\section{Resumo}

As demandas por tratamentos odontológicos para recessões gengivais estão aumentando cada dia mais, o comprometimento estético e a hipersensibilidade causada por essa alteração no periodonto é algo que causa muito incômodo na população de forma geral. Apesar de existir uma maior frequência em adultos, alguns estudos têm demonstrado a ocorrência em criança. O objetivo deste trabalho foi realizar uma revisão narrativa e atual dos últimos 10 anos sobre os fatores que podem contribuir par recessão gengival em crianças. Buscas distintas foram realizadas na base de dados Pubmed/Medline. Os critérios de elegibilidade foram: estudos publicados na língua inglesa, recessão gengival, doença mucogengival, etiologia, prevalência em crianças, estudo de coorte, longitudinal e casos clínicos. Como critérios de exclusão foi idade (casos acima de 19 anos), revisão de literatura, textos fora do tema. Do total dos textos encontrados, apenas 9 cumpriram os critérios de inclusão. Concluímos que não houve, nestes últimos 10 anos, pesquisas sobre o tema na dentição decídua. As manifestações aqui descritas como: bruxismo, tratamento ortodôntico, doenças sistêmicas, maloclusões, freios labiais estavam relacionados em crianças, porém, na fase dentadura mista e ou permanentes.

Palavras-chave: Retração gengival; Odontopediatria; Gengiva.

\begin{abstract}
Demands for dental treatments for gingival recessions are increasing every day, the aesthetic compromise and hypersensitivity caused by this change in the periodontium is something that causes a lot of discomfort in the population in general. Although there is a greater frequency in adults, some studies have shown that it occurs in children. The objective of this work was to carry out a narrative and current review of the last 10 years about the factors that may contribute to gingival recession in children. Different searches were performed in the Pubmed/Medline database. Eligibility criteria were: studies published in English, gingival recession, mucogingival disease, etiology, prevalence in children, cohort study, longitudinal and clinical cases. Exclusion criteria were age (cases over 19 years old), literature review, texts outside the topic. Of the total texts found, only 9 met the inclusion criteria. We conclude that, in the last 10 years, there has been no research on the topic of primary dentition. The manifestations described here as: bruxism, orthodontic treatment, systemic diseases, malocclusions, labial frenulum were related in children, however, in mixed and/or permanent dentition phase.
\end{abstract}

Keywords: Gingival recession; Paediatric dentistry; Gingiva.

\section{Resumen}

La demanda de tratamientos dentales para las recesiones gingivales es cada día mayor, el compromiso estético y la hipersensibilidad provocada por este cambio en el periodonto es algo que provoca mucho malestar en la población en 
general. Aunque existe una mayor frecuencia en adultos, algunos estudios han demostrado que ocurre en niños. El objetivo de este trabajo fue realizar una revisión narrativa y actual de los últimos 10 años sobre los factores que pueden contribuir a la recesión gingival en los niños. Se realizaron diferentes búsquedas en la base de datos Pubmed / Medline. Los criterios de elegibilidad fueron: estudios publicados en inglés, recesión gingival, enfermedad mucogingival, etiología, prevalencia en niños, estudio de cohorte, casos longitudinales y clínicos. Los criterios de exclusión fueron edad (casos mayores de 19 años), revisión de la literatura, textos fuera del tema. Del total de textos encontrados, solo 9 cumplieron los criterios de inclusión. Concluimos que, en los últimos 10 años, no se ha realizado ninguna investigación sobre el tema de la dentición temporal. Las manifestaciones aquí descritas como: bruxismo, tratamiento de ortodoncia, enfermedades sistémicas, maloclusiones, frenillo labial se relacionaron en niños, sin embargo, en fase de dentición mixta y / o permanente.

Palabras clave: Recesión gingival; Odontología pediátrica; Encía.

\section{Introdução}

A manutenção da saúde bucal compreende a saúde dos tecidos periodontais. Assim, é fundamental que odontopediatra reconheça os padrões de normalidades, incluindo suas variações fisiológicas para que seja possível o reconhecimento das alterações patológicas no periodonto. O periodonto é o conjunto de tecidos que protegem e sustentam os dentes na cavidade bucal e forma unidade de desenvolvimento, biológica e funcional que sofre alterações de acordo com a faixa etária (Slots, 2017). Quando pensamos na primeira infância, o periodonto sofre modificações devido à erupção dos dentes decíduos (Bimstein \& Matsson, 1999). Em virtude das constantes modificações que ocorrem em função da idade (exfoliação e erupção dentária), é uma tarefa difícil estabelecer um quadro específico dos dados clínicos de normalidade gengival em criança. A gengiva é definida como a parte da mucosa mastigatória que cobre o processo alveolar e circunda a porção cervical dos dentes. A doença periodontal mais comum em crianças é a gengivite e o seu tratamento consiste com alto grau de evidência, em controle do biofilme dentário. Entretanto, embora a condição seja prevalente e o tratamento baseado em forte evidência, a importância da atenção a essa condição nessa faixa etária ainda não é clara (Alrayyes \& Hart, 2011; Tsami et al., 2010).

Os dados de um estudo epidemiológico longitudinal demonstraram que em uma população da Europa Ocidental, mesmo recebendo atendimento odontológico regular com um nível razoável de higiene oral, as recessões gengivais foram encontradas em mais de $60 \%$ da população mais jovem (ou seja, até 20 anos de idade) e em mais de 90\% no grupo etário acima dos 50 anos mais. No entanto, a ocorrência de recessões gengivais foi significativamente maior em um população sem nenhum atendimento odontológico (Loe et al., 1992).

As recessões gengivais se caracterizam clinicamente pelo deslocamento da gengiva marginal livre em posição mais apical à junção cemento-esmalte, e que pode estar localizada em um dente ou generalizada por toda boca. A prevalência de recessão gengival depende do sexo e da população e aumenta com a idade do indivíduo. Sendo mais frequente em adultos, e pouco evidenciada na dentição decídua, com uma média de porcentagem de $8 \%$ em crianças com idade entre 8 e 12 anos (Younes \& El Angbawi, 1983). As superfícies vestibulares dos incisivos centrais inferiores e molares superiores são os locais mais frequentemente (Khocht et al., 1993). Essa mudança da gengiva marginal pode trazer danos estéticos e até mesmo funcionais aos pacientes, uma vez que normalmente são associadas a presença de cárie radicular, abrasão cervical e hipersensibilidade dentinária (Song, 2013).

A etiologia das recessões gengivais é considerada multifatorial entre eles estão os fatores de desenvolvimento (fenestração do osso alveolar, erupção dentária ectópica ou posição na arcada dentária) e / ou fatores adquiridos (lesão, perfuração ou escovação dentária com força, falta de higienização) são os fatores considerados determinantes para o surgimento dessa patologia (Camargo et al., 2019). Além disso, o biofilme dentário associada a outros fatores predisponentes como freios e bridas, oclusão traumatogênica, mau posicionamento dentário, ausência de gengiva inserida e fenótipo periodontal fino, também, podem contribuir para o seu desenvolvimento. Dessa forma é fundamental detectar a presença desses fatores causais e/ou predisponentes para pelo menos impedir a progressão das recessões (Cortellini \& Bissada, 2018; 
Joss-Vassalli et al., 2010).(Halai et al., 2020)

Na odontopediatria, a recessão gengival ao redor de um ou mais dentes é sempre um motivo de preocupação. Nesses casos, a gengiva pode estar inflamada, ou não, dependendo da presença ou ausência do biofilme dentário. A etiologia mais frequênte da recessão gengival em criança é a posição dos dentes na arcada. Dentes vestibularizados, inclinados ou girovertidos, de tal forma que as raízes são projetadas para vestibular pode ser um fator predisponente para tal ocorrência que pode ser transitória, durante a erupção dentária, corrigindo por si só quando os dentes atingirem seu alinhamento definitivo (Renkema et al., 2015; Zhu \& Chen, 2013). Outros fatores como alveólise, hábitos parafuncionais, trauma dentário, escovação traumática ou à presença de freios e bridas patológicas, devem ser diagnosticadas e tratados o mais precocemente possível durante a dentição decídua (Camargo et al., 2019; Nwhator, 2014). Nestes casos, é essencial o tratamento baseado nos procedimentos básicos periodontais, motivar a higiene bucal e quando necessário realização da cirurgia. Por ser um assunto pouco discutido na odontopediatria clínica, o objetivo deste trabalho foi realizar uma revisão narrativa e atual das possíveis etiologia da recessão gengival observada em crianças.

\section{Metodologia}

Trata-se de uma revisão integrativa com coleta de dados realizada através de levantamentos bibliográficos. Inicialmente, foi adotada a seguinte questão norteadora: quais fatores que podem contribuir para recessão na dentição decídua?

Para responder a essa pergunta, foram realizadas revisões da literatura, buscando reunir e sintetizar os resultados encontrados nos estudos primários dos últimos 10 anos, que analisaram a relação entre a recessão gengiva na dentição decídua. Para isso, foram utilizados artigos científicos coletados nas bases de dados do Medline/Pubmed (Estrela, 2018; Ludke \& Andre, 2013). Os descritores foram escolhidos por sua importância na delimitação do tema de acordo com os termos de Medical Subject Headings (MeSH):

Medline/Pubmed: ((Mucogingival disease or Gingival Recession) AND (children or preeschool)) AND (etiology or frequency or prevalence) Filters: from 2011 - 2021

Após a busca, foi realizada a triagem e coleta de dados. Os títulos e resumos foram verificados quanto aos critérios de inclusão: estudos publicados na língua inglesa, recessão gengival, doença mucogengival, etiologia, prevalência em crianças, estudo de coorte, longitudinal e casos clínicos. Como critérios de exclusão foi idade (casos acima de 19 anos), revisão de literatura, textos fora do tema. As etapas de extração e análise dos resultados dos estudos primários foram realizadas por dois revisores. A pesquisa no site de busca pubmed foi salva e os artigos encontrados $(n=45)$ foram armazenados para leitura no aplicativo Rayyan. Os dados coletados de cada artigo incluído foram: ano, autores, país, título, idade das crianças e conclusão. Os resultados foram analisados de maneira descritiva.

\section{Resultados e Discussão}

Após a consulta na base de dados, foram identificados 45 artigos potencialmente elegíveis, deste total, 36 foram excluídos após a leitura do título e resumo por estarem fora do tema ou por não abordar a recessão gengival em crianças, totalizando 9 artigos para leitura na íntegra. 
Quadro 1: Consolidação dos artigos dessa revisão narrativa.

\begin{tabular}{|c|c|c|c|c|c|c|}
\hline & Autor & Ano & País & Título & Amostra & Conclusão \\
\hline 1 & Blignaut et al., & 2019 & Africa do Sul & $\begin{array}{l}\text { Gingival Recession and Localized } \\
\text { Aggressive Periodontitis Among } \\
\text { HIV-infected Children and } \\
\text { Adolescents Receiving } \\
\text { Antiretroviral Therapy }\end{array}$ & $\begin{array}{l}554 \text { crianças de } \\
5-19 \text { anos }\end{array}$ & $\begin{array}{l}14 \% \text { apresentaram recessão } \\
\text { gengival. É importante o } \\
\text { acompanhamento odontologico de } \\
\text { crianças com doenças sistêmicas. }\end{array}$ \\
\hline 2 & Mijuskovic & 2018 & $\begin{array}{l}\text { República } \\
\text { Tcheca }\end{array}$ & $\begin{array}{l}\text { Tooth wear and gingival } \\
\text { recession in } 210 \text { orthodontically } \\
\text { treated patients: a retrospective } \\
\text { cohort study }\end{array}$ & 210 crianças & $\begin{array}{l}\text { Dentes com desgaste dentário de } \\
\text { grau moderado ou grave têm } 23 \% \\
\text { mais chances de desenvolver } \\
\text { recessão gengival. }\end{array}$ \\
\hline 3 & Zhu & 2013 & China & $\begin{array}{l}\text { Association between gingival } \\
\text { recession and proclination of } \\
\text { maxillary central incisors near the } \\
\text { cleft in patients with unilateral } \\
\text { cleft lip and palate: A } \\
\text { retrospective case-control study }\end{array}$ & $\begin{array}{l}40 \text { crianças } \\
\text { com media de } \\
\text { idade de } 13.2 \\
\text { anos }\end{array}$ & $\begin{array}{l}\text { A inclinação do incisivo central } \\
\text { superior adjacente à fenda está } \\
\text { positivamente correlacionada com a } \\
\text { recessão gengival assim como o } \\
\text { plano sela nasio pode também ser } \\
\text { um fator predisponente. }\end{array}$ \\
\hline 4 & Gebistorf & 2018 & Suiça & $\begin{array}{l}\text { Gingival recession in orthodontic } \\
\text { patients } 10 \text { to } 15 \text { years } \\
\text { posttreatment: A retrospective } \\
\text { cohort study }\end{array}$ & $\begin{array}{l}44 \text { crianças } \\
\text { com média de } \\
\text { idade de } 12.1 \\
\text { anos }\end{array}$ & $\begin{array}{l}\text { A recessão gengival aumentou } \\
\text { durante o tratamento ortodôntico. }\end{array}$ \\
\hline 5 & Renkema & 2014 & Suiça & $\begin{array}{l}\text { Gingival labial recessions and the } \\
\text { post-treatment proclination of } \\
\text { mandibular incisors }\end{array}$ & $\begin{array}{l}117 \text { crianças } \\
\text { com idade } \\
\text { media de } 12 \\
\text { anos }\end{array}$ & $\begin{array}{l}\text { A proclinação dos incisivos } \\
\text { inferiores não aumentou o risco de } \\
\text { desenvolvimento de recessão } \\
\text { gengival durante a observação de } \\
\text { cinco anos em comparação aos } \\
\text { dentes não proclinados. }\end{array}$ \\
\hline 6 & Nosrati & 2013 & $\begin{array}{l}\text { Estados } \\
\text { Unidos }\end{array}$ & $\begin{array}{l}\text { Gingival evaluation of the } \\
\text { pediatric cardiac patient }\end{array}$ & $\begin{array}{l}50 \text { crianças } \\
\text { entre } 7 \text { a } 13 \\
\text { anos }\end{array}$ & $\begin{array}{l}\text { Crianças com doenças } \\
\text { cardiovasculares congênitas } \\
\text { apresentaram maior prevalência de } \\
\text { doença periodontal, evidenciada por } \\
\text { gengivite, placa, cálculo e recessão. }\end{array}$ \\
\hline 7 & Renkema & 2013 & Suiça & $\begin{array}{l}\text { Development of labial gingival } \\
\text { recessions in orthodontically } \\
\text { treated patients }\end{array}$ & $\begin{array}{l}302 \text { crianças } \\
\text { com idade } \\
\text { média de } 13.6 \\
\text { anos }\end{array}$ & $\begin{array}{l}\text { A prevalência de recessões } \\
\text { gengivais aumenta continuamente } \\
\text { após o tratamento ortodôntico. } \\
\text { Nenhuma variável, exceto a idade ao } \\
\text { final do tratamento, parece estar } \\
\text { associada ao desenvolvimento de } \\
\text { recessões gengivais. }\end{array}$ \\
\hline 8 & Nwhator & 2014 & Nigéria & $\begin{array}{l}\text { Gingival recession in a child- } \\
\text { patient; easily missed etiologies: } \\
\text { case report with video }\end{array}$ & $\begin{array}{l}\text { criança de } 9 \\
\text { anos }\end{array}$ & $\begin{array}{l}\text { A etiologia da recessão gengival } \\
\text { neste paciente foi freio labial } \\
\text { inferior alto, sobremordida } \\
\text { traumática e bruxismo. }\end{array}$ \\
\hline 9 & Incerti-Parenti & 2015 & $\begin{array}{l}\text { Não } \\
\text { mencionado }\end{array}$ & $\begin{array}{l}\text { Periodontal Side Effect During } \\
\text { Orthopedic Face Mask Therapy }\end{array}$ & $\begin{array}{l}\text { criança de } 7 \\
\text { anos }\end{array}$ & $\begin{array}{l}\text { apresentou recessão gengival do } \\
\text { dente } 41 \text { e acúmulo de placa severa } \\
\text { após } 3 \text { meses de terapia com } \\
\text { máscara facial. A melhora decisiva } \\
\text { começou quando a extensão vertical } \\
\text { da almofada do queixo foi reduzida } \\
\text { para evitar pressão na área afetada. }\end{array}$ \\
\hline
\end{tabular}

Fonte: Autores. 
O tipo de estudo mais prevalente foi longitudinal e 2 artigos foram do tipo de caso clínico. Nenhum artigo foi realizado no Brasil, já na Suíça houveram 3 artigos nos outros artigos, os trabalhos foram realizados na África do Sul, China, República Tcheca, Nigéria, Estados Unidos e um artigo não foi mencionado a localização.

Os fatores etiológicos para ocorrência de recessão gengival estavam relacionados ao tratamento ortodôntico, doenças sistêmicas que afetam o periodonto e fatores como trauma e bruxismo.

Apesar da quantidade limitada de estudos incluídos nesta revisão narrativa, há uma clara relação dos fatores locais para a ocorrência de recessão gengival, confirmando esses fatores preditivos já apresentados na literatura. Isso porque, de acordo com a literatura revisada, a recessão gengival apresenta etiologia multifatorial tais como técnicas de escovação falhas, mau posicionamento dental, inflamação gengival, determinados movimentos ortodônticos e inserção alta de freios e bridas (Alrayyes \& Hart, 2011; Song, 2013; Tsami et al., 2010; Vasconcelos et al., 2012; Younes \& El Angbawi, 1983; Zhu \& Chen, 2013). Portanto, é unanime nos autores sobre a importância da observação clínica do freio labial inferior e inserções próximas às gengivas marginal e papilar porque podem provocar retrações gengivais (Nwhator, 2014; Renkema et al., 2015; Zhu \& Chen, 2013). Entretanto não foi observado nenhum trabalho relacionando a recessão gengival na dentição decídua. Todos os trabalhos aqui incluídos, as crianças estavam com dentição mista. Porém, a recessão gengival também pode ser observada na dentição decídua. A presença de problemas mucogengivais em crianças na primeira infância não é freqüente, mas quando presentes na dentição decídua, as recessões gengivais estão principalmente associadas a hábitos bucais, como sucção de chupeta e digital, e padrão atípico de deglutição com interposição lingual que conduz muitas vezes à alteração na posição dos dentes anteriores (Machtei et al., 1990). Porém, independente de ser na dentição decídua ou permanente, a recessão gengival dentária é sempre um motivo de preocupação. Devido à exposição do cemento, essa alteração pode trazer como conseqüências maior susceptibilidade à cárie radicular, hipersensibilidade resultante de uma reação pulpar à agressão da dentina além do comprometimento estético. O paciente pode desenvolver sintomatologia dolorosa, levando a negligenciar os princípios básicos da higiene bucal, podendo surgir quadros de cárie e doença periodontal (Alrayyes \& Hart, 2011).

Dentre os artigos incluídos, dois estavam relacionando a recessão gengival com pacientes que apresentavam doenças sistêmicas (Blignaut et al., 2019; Nosrati et al., 2013). O tratamento da doença periodontal em crianças com doenças sistêmicas é um desafio. É importante que o diagnóstico correto seja feito para o tratamento adequado a ser administrado. Um histórico médico e odontológico completo deve ser feito e a consulta com um médico é necessária para um melhor tratamento possível. Muitas condições que apresentam a perda precoce do dente decíduo são sérias e, criança que apresenta perda inexplicável de dentes, requer avaliação multidisciplinar imediatas, como nos casos de doença de Chediak-Higashi, HIV, Síndrome de papillom-Lefèvre, diabetes (Blignaut et al., 2019; Halai et al., 2020; Novotna et al., 2015; Rezende et al., 2013), Portanto, toda e qualquer alteração metabólica que caracterize uma doença sistêmica ou modificações transitórias tem a possibilidade de interferir na progressão da doença periodontal (Song, 2013).

Quando os estudos sobre o tratamento ortodôntico e recessão gengival há uma longa história de controvérsia em torno de quando corrigir essas recessões gengivais e no que diz respeito às correções de tempo com terapias ortodônticas (Gebistorf et al., 2018; Incerti-Parenti et al., 2015; Renkema et al., 2015). Uma comunicação próxima entre o odontopediatra, o ortodontista e o periodontista é um passo crucial na determinação o prognóstico dos dentes recuados. O bom senso também pode ser uma ótima ferramenta. O mais importante é que o paciente tenha acompanhamento longitudinal uma vez que a avaliação a longo prazo do tratamento ortodôntico não mostra nenhum efeito perceptível na saúde periodontal posterior (Vasconcelos et al., 2012). Por fim, desgastes dentários como o bruxismo também pode ser um fator etiológico para ocorrência de recessão gengival (Mijuskovic et al., 2018; Nwhator, 2014).

Conhecendo a etiologia da recessão gengival pode se optar para melhor tratamento em cada caso. Assim, quando a etiologia está associada ao freio patológico, durante a dentição decídua, o tratamento é realizar os procedimentos básicos 
periodontais, motivar a higienização bucal correta e ter uma abordagem de equipe deve ser considerada para o sucesso no tratamento de crianças. A comunicação próxima com odontopediatras e outros especialistas, como ortodontistas, endodontistas e cirurgiões orais, é a chave para um resultado bem-sucedido.

\section{Considerações Finais}

Nota-se que na literatura não houve, nestes últimos 10 anos, pesquisas quais fatores poderiam ocasionar recessão gengival na dentição decídua. As manifestações aqui descritas como: bruxismo, tratamento ortodôntico, doenças sistêmicas, maloclusões, freios labiais estavam relacionados em crianças porém em fase dentadura mista.

Deixamos aqui aberto a sugestão de pesquisas clínicas futuras do tipo de prevalência, distribuição e possíveis etiologias sobre esse tema para que pudesse guiar o odontopediatra uma atenção especial nesta alteração mucogengival em crianças.

\section{Referências}

Alrayyes, S., \& Hart, T. C. (2011, Apr). Periodontal disease in children. Dis Mon, 57(4), 184-191. https://doi.org/10.1016/j.disamonth.2011.03.004

Bimstein, E., \& Matsson, L. (1999, May-Jun). Growth and development considerations in the diagnosis of gingivitis and periodontitis in children. Pediatr Dent, 21(3), 186-191. https://www.ncbi.nlm.nih.gov/pubmed/10355010

Blignaut, E., Rossouw, T. M., Becker, P. J., Mavuso, D. S., \& Feucht, U. D. (2019, Jun). Gingival Recession and Localized Aggressive Periodontitis Among HIV-infected Children and Adolescents Receiving Antiretroviral Therapy. Pediatr Infect Dis J, 38(6), e112-e115. https://doi.org/10.1097/INF.0000000000002166

Camargo, F. C. D., Imparato, J. C. P., \& Rezende, K. M. P. e. C. (2019). Alveólise em dente decíduo- relato de caso Revista de Odontopediatría Latinoamericano, 9(2), 7. https://doi.org/https://doi.org/10.47990/alop.v9i2.178

Cortellini, P., \& Bissada, N. F. (2018, Jun). Mucogingival conditions in the natural dentition: Narrative review, case definitions, and diagnostic considerations. J Periodontol, 89 Suppl 1, S204-S213. https://doi.org/10.1002/JPER.16-0671

Estrela, C. (2018). Metodologia cientifica: ciênica, ensino, pesquisa A. Médicas, Ed.).

Gebistorf, M., Mijuskovic, M., Pandis, N., Fudalej, P. S., \& Katsaros, C. (2018, May). Gingival recession in orthodontic patients 10 to 15 years posttreatment: A retrospective cohort study. Am J Orthod Dentofacial Orthop, 153(5), 645-655. https://doi.org/10.1016/j.ajodo.2017.08.020

Halai, H., Somani, C., Donos, N., \& Nibali, L. (2020, Jun). Periodontal status of children with primary immunodeficiencies: a systematic review. Clin Oral Investig, 24(6), 1939-1951. https://doi.org/10.1007/s00784-019-03055-Z

Incerti-Parenti, S., Checchi, V., Molinari, C., \& Alessandri-Bonetti, G. (2015, Winter). Periodontal Side Effect During Orthopedic Face Mask Therapy. Int J Orthod Milwaukee, 26(4), 49-51. https://www.ncbi.nlm.nih.gov/pubmed/27029093

Joss-Vassalli, I., Grebenstein, C., Topouzelis, N., Sculean, A., \& Katsaros, C. (2010, Aug). Orthodontic therapy and gingival recession: a systematic review. Orthod Craniofac Res, 13(3), 127-141. https://doi.org/10.1111/j.1601-6343.2010.01491.x

Khocht, A., Simon, G., Person, P., \& Denepitiya, J. L. (1993, Sep). Gingival recession in relation to history of hard toothbrush use. J Periodontol, 64(9), 900905. https://doi.org/10.1902/jop.1993.64.9.900

Loe, H., Anerud, A., \& Boysen, H. (1992, Jun). The natural history of periodontal disease in man: prevalence, severity, and extent of gingival recession. $J$ Periodontol, 63(6), 489-495. https://doi.org/10.1902/jop.1992.63.6.489

Ludke, M., \& Andre, M. E. D. A. (2013). Pesquisas em educação: uma abordagem qualitativa.(E.P.U., Ed.).

Machtei, E. E., Zubery, Y., Bimstein, E., \& Becker, A. (1990, Dec). Anterior open bite and gingival recession in children and adolescents. Int Dent J, 40(6), 369-373. https://www.ncbi.nlm.nih.gov/pubmed/2276836

Mijuskovic, M., Gebistorf, M. C., Pandis, N., Renkema, A. M., \& Fudalej, P. S. (2018, Jul 27). Tooth wear and gingival recession in 210 orthodontically treated patients: a retrospective cohort study. Eur J Orthod, 40(4), 444-450. https://doi.org/10.1093/ejo/cjx083

Nosrati, E., Eckert, G. J., Kowolik, M. J., Ho, J. G., Schamberger, M. S., \& Kowolik, J. E. (2013, Sep-Oct). Gingival evaluation of the pediatric cardiac patient. Pediatr Dent, 35(5), 456-462. https://www.ncbi.nlm.nih.gov/pubmed/24290561

Novotna, M., Podzimek, S., Broukal, Z., Lencova, E., \& Duskova, J. (2015). Periodontal Diseases and Dental Caries in Children with Type 1 Diabetes Mellitus. Mediators Inflamm, 2015, 379626. https://doi.org/10.1155/2015/379626 
Research, Society and Development, v. 10, n. 14, e541101422459, 2021

(CC BY 4.0) | ISSN 2525-3409 | DOI: http://dx.doi.org/10.33448/rsd-v10i14.22459

Nwhator, S. (2014, Mar). Gingival recession in a child-patient; easily missed etiologies: case report with video. Ann Med Health Sci Res, 4(Suppl 1), S18-21. https://doi.org/10.4103/2141-9248.131698

Renkema, A. M., Navratilova, Z., Mazurova, K., Katsaros, C., \& Fudalej, P. S. (2015, Oct). Gingival labial recessions and the post-treatment proclination of mandibular incisors. Eur J Orthod, 37(5), 508-513. https://doi.org/10.1093/ejo/cju073

Rezende, K. M., Canela, A. H., Ortega, A. O., Tintel, C., \& Bonecker, M. (2013, Nov-Dec). Chediak-Higashi syndrome and premature exfoliation of primary teeth. Braz Dent J, 24(6), 667-670. https://doi.org/10.1590/0103-6440201302258

Slots, J. (2017, Oct). Periodontitis: facts, fallacies and the future. Periodontol 2000, 75(1), 7-23. https://doi.org/10.1111/prd.12221

Song, H. J. (2013, Jan). Periodontal considerations for children. Dent Clin North Am, 57(1), 17-37. https://doi.org/10.1016/j.cden.2012.09.009

Tsami, A., Petropoulou, P., Panayiotou, J., Mantzavinos, Z., \& Roma-Giannikou, E. (2010, Sep). Oral hygiene and periodontal treatment needs in children and adolescents with coeliac disease in Greece. Eur J Paediatr Dent, 11(3), 122-126. https://www.ncbi.nlm.nih.gov/pubmed/21080751

Vasconcelos, G., Kjellsen, K., Preus, H., Vandevska-Radunovic, V., \& Hansen, B. F. (2012, Jan). Prevalence and severity of vestibular recession in mandibular incisors after orthodontic treatment. Angle Orthod, 82(1), 42-47. https://doi.org/10.2319/021411-108.1

Younes, S. A., \& El Angbawi, M. F. (1983, Aug). Gingival recession in the mandibular central incisor region of Saudi schoolchildren aged 10-15 years. Community Dent Oral Epidemiol, 11(4), 246-249. https://doi.org/10.1111/j.1600-0528.1983.tb01886.x

Zhu, S., \& Chen, Z. (2013, Mar). Association between gingival recession and proclination of maxillary central incisors near the cleft in patients with unilateral cleft lip and palate: A retrospective case-control study. Am J Orthod Dentofacial Orthop, 143(3), 364-370. https://doi.org/10.1016/j.ajodo.2012.10.021 